\title{
Quand les morts de Lampedusa entrent en politique : damnatio memorice
}

When the Dead of Lampedusa Become Political: Damnatio Memoriae

\section{Évelyne Ritaine}

\section{(2) OpenEdition \\ Journals}

Édition électronique

URL : http://journals.openedition.org/conflits/19110

DOI : $10.4000 /$ conflits. 19110

ISSN : $1777-5345$

Éditeur :

CECLS - Centre d'études sur les conflits - Liberté et sécurité, L'Harmattan

Édition imprimée

Date de publication : 31 décembre 2015

Pagination : 117-142

ISBN : 978-2-343-08728-3

ISSN : 1157-996X

Référence électronique

Évelyne Ritaine, "Quand les morts de Lampedusa entrent en politique : damnatio memoriæ », Cultures \& Conflits [En ligne], 99-100 | automne/hiver 2015, mis en ligne le 16 février 2017, consulté le 31 mars 2021. URL : http://journals.openedition.org/conflits/19110; DOI : https://doi.org/10.4000/conflits. 19110 


\section{Quand les morts de Lampedusa entrent en politique : damnatio memoriæ}

\section{Évelyne RITAINE}

Évelyne Ritaine est Directrice de recherche FNSP, Centre Émile Durkheim, Sciences Po Bordeaux. Sur les frontières et les migrations, elle a notamment dirigé l'onvrage: L'Europe du sud face à l'immigration. Politique de l'Étranger, Paris, PUF, 2005, et publié : "La barrière et le checkpoint: mise en politique de l'asymétrie ", Cultures \& Conflits, 73, 2009, pp. 13-33; "Dramaturgie de l'intrusion migratoire : teatro all'italiana ", in D.-C. Martin (ed.), L'identité en jeux. Pouvoirs, identifications, mobilisations, Paris, Karthala, 2010, pp. 201-221; La fabrique politique d'une frontière européenne en Méditerranée. Le “jeu du mistigri” entre les États et l'Union, Paris, Les Etudes du CERI, 186, 2012. Elle anime un carnet de recherche en ligne consacré aux frontières blindées : http://enigmur.hypotheses.org/

\section{"Li lasciamo annegare, per negare», Erri De Luca}

$\mathrm{D}$ ans la Rome antique, la damnatio memorice (la condamnation de la mémoire) effaçait tout souvenir et toute trace de personnes considérées comme ayant porté atteinte à l'honneur de la cité et condamnées à mort : comme si elles n'avaient jamais existé. Dans l'Union Européenne (UE) aujourd'hui, il se passe quelque chose de comparable, avec l'acceptation tacite de la mort de milliers de migrants aux portes de l'Europe, perdus corps et biens, sans laisser de trace : comme s'ils n'avaient jamais existé. Comment cet effacement est-il possible, moralement et politiquement ? Par quelles médiations et par quelles interprétations cet effacement est-il produit ? 1

Le lieu emblématique de ce drame et de cet effacement est une petite île italienne, Lampedusa, perdue au milieu de la Méditerranée centrale, entre

1. Cette recherche a bénéficié de la collaboration de Stéphanie Bonnefille (maitresse de conférences en linguistique cognitive, Climas, Université Bordeaux 3) qui a effectué une analyse linguistique des principaux discours publics tenus à propos de cet événement. Elle a aussi complété notre fonds iconographique par un reportage photographique à Lampedusa. Je tiens à remercier, pour leur apport à cette réflexion, Paolo Cuttitta, Mathias Delori, Bachir Khalef, les membres de l'équipe Médimurs, ainsi que mes relecteurs anonymes. 
Sicile et Tunisie. Située sur la route migratoire la plus mortelle du monde 2 , marquée par des arrivées et des noyades continues depuis les années 1990, et par des naufrages dramatiques, en octobre 2013 et en février 2015 notamment, son nom est devenu synonyme d'un scandale moral et politique au seuil de l'UE. Car il y a comme un paradoxe Lampedusa : son éloignement semblait propice pour reléguer sur l'île les difficultés liées à la migration irrégulière ${ }^{3}$; la théâtralité insulaire ${ }^{4}$ a, au contraire, permis la mise en évidence, par l'exposition médiatique et par les actions militantes, de la puissance létale de la règle européenne. Sur ce lieu frontière, en effet, se matérialise, aux yeux de tous, le pouvoir moderne « de faire vivre et de laisser mourir », tel que M. Foucault l'a théorisé ${ }^{5}$. Au-delà des préoccupations pastorales de l'État moderne (protection de la population intérieure, par le contrôle de la frontière, et respect des droits humains des entrants, fussent-ils irréguliers), il s'agit, plus précisément, $\mathrm{du}$ "pouvoir d'exposer à la mort ${ }^{6}$ ", dans une "nécropolitique » de la frontière qui «tolère des pertes 7 ». Les corps des disparus en mer sont des marqueurs de la frontière, ils témoignent de la puissance de la souveraineté, et en même temps attestent de la violence politique d'un « espace d'exception en mer ${ }^{8} »$ : disparus sans laisser de traces, ou cadavres retrouvés en mer, les corps migrants sont la trace dérangeante $\mathrm{du}$ « deadly prevented from entering ${ }^{9}$ ».

Longtemps, les disparitions en Méditerranée ont été ignorées ou négligées : elles étaient des non-événements. La presse, comme les travaux de recherche, les évoquaient dans l'abstraction des statistiques, ou bien dans le

2. Selon le Haut-commissariat des Nations unies pour les réfugiés, cité dans « La traversée de la Méditerranée, route la plus mortelle du monde », Le Monde, 10 décembre 2014. Lampedusa est à $205 \mathrm{~km}$ de la Sicile, $167 \mathrm{~km}$ de la Tunisie, $355 \mathrm{~km}$ de la Libye. Entre 2000 et 2013, il y a eu 6400 disparus sur la route de Lampedusa, 8000 disparus en prenant en compte l'ensemble du Détroit de Sicile.

3. On s'en tient ici, suivant en cela la plupart des experts de la question migratoire, au terme le moins stigmatisant et le plus général : migration « irrégulière » plutôt que « clandestine » ou «illégale ».

4. Pour la généalogie de la «frontiériation» de Lampedusa, voir l'article de Paolo Cuttitta dans ce numéro, ainsi que : Cuttitta P., Lo spettacolo del confine. Lampedusa tra produzione e messa in scena della frontiera, Milano, Mimesis (Eterotopie), 2012 ; “Borderizing' the Island. Setting and Narratives of the Lampedusa 'Border Play"”, ACME, 2014, 13/2, pp. 196-219; "La "frontiérisation” de Lampedusa, comment se construit une frontière ", L'Espace Politique, 25, 2015 [https://espacepolitique.revues.org/3336].

5. Foucault M., Il faut défendre la société, Paris, Gallimard/Seuil, 1997.

6. Mbembe A., "Nécropolitique », Raisons politiques, 2006, 21, pp. 29-60, p. 30 ; voir aussi Khosvari S., "Illegal Traveller". An Auto-ethnography of Borders, Basingstoke, New-York, Palgrave-Macmillan, 2010 ; Bauman Z., Vies perdues. La modernité et ses exclus, Paris, Payot, 2006.

7. Inda J.X., Targeting Immigrants: Government, Technology, and Ethics, Oxford, Blackwell, 2006. Dans l'ensemble du texte toutes les traductions sont de l'auteure.

8. Perera S., “They Give Evidence': Bodies, Borders and the Disappeared”, Social Identities: Journal for the Study of Race, Nation and Culture, 12, 6, 2006, pp. 637-656.

9. Littéralement : "empêché d'entrer par le risque de mort ", Albahari M., Death and the Moral State: Making Borders and Sovereignty at the Southern Edges of Europe, Working Paper 136, CCIS University of California, San Diego, 2006, p. 27 ; voir aussi Albahari M., Crimes of Peace: Mediterranean Migrations at the World's Deadliest Border, Philadelphie, University of Pennsylvania Press, 2015. 
langage littéraire de la non-existence : fantômes, disparus, limbes, etc. Ainsi désignées, elles représentent la fin de la "vie nue 10 ", celle qui est déréalisée, anonyme, celle qui disparait aussi, très vite, dans les discours publics. Lorsque, au contraire, comme c'est le cas à Lampedusa en 2013, les corps ou les traces des noyés apparaissent, ils sont la marque irréfutable, par leurs possibles identification et reconnaissance, de la violence politique de la frontière. Ces traces identifiables attestent de la puissance létale de la frontière et sont si politiquement insupportables qu'il n'est pas possible de ne pas en donner une ou des interprétations politiques. Un travail politique se déploie alors pour interpréter ces morts, dans des versions différentes, et pour affronter l'enjeu fondamental de leur cadrage cognitif et politique : sont-ils réduits à l'invisibilité ou peuvent-ils être rendus visibles ? Sont-ils condamnés à l'anonymat ou peut-on leur reconnaitre une identité ? Quels sont les effets politiques des réponses à ces questions?

Lorsque le gouvernement de S. Berlusconi avait choisi de faire de Lampedusa le principal centre de triage des migrants arrivant par mer, il s'agissait pour le pouvoir politique italien de créer un point de fixation des problèmes de la migration irrégulière, dans un espace restreint et lointain. Cette stratégie semble avoir eu, avec le temps, l'effet inverse : c'est bien à Lampedusa qu'a été rendu évident, aux yeux du monde entier, le péril des tentatives de passage par mer. La concentration d'événements dramatiques autour de ce théâtre qu'est l'île a eu un effet inouï. Avant tout traitement politique et bureaucratique, la réalité de la migration irrégulière y apparaît en effet crûment : tout ce qui est invisible ailleurs est ici visible. En effet, l'invisibilité des migrants disparus en mer est, la plupart du temps, une invisibilité radicale : la migration peut se solder par une disparition corps et biens et identité. Aussi quand E. De Luca écrit «Li lasciamo annegare, per negare 11 » (littéralement: "Nous les laissons se noyer, pour nier »), il pose l'invisibilité de certaines morts, et donc de certaines vies. Car, pour reprendre les mots de J. Butler, «si une vie ne peut être pleurée, elle n'est pas tout à fait une vie; elle n'a pas valeur de vie et ne mérite pas qu'on la remarque 12 ». Les migrants disparus en mer ne sont, a priori, que des nombres : longtemps ignorées, souvent sous-estimées, ces disparitions réduisent les migrants à des unpersons ${ }^{13}$, des personnes qui n'existent pas et n'ont jamais existé.

10. Agamben G., Homo sacer. Le powvoir souverain et la vie nue, Paris, Seuil, 1997.

11. De Luca E., " Noi, carcerieri di viaggiatori ", libreidee.org, 23 mars 2010 [http://www.libreidee.org].

12. Butler J., Vie précaire, Les pouvoirs du denil et de la violence après le 11 septembre 2001, Paris, Paris, Éditions Amsterdam, 2005, p. 62.

13. Orwell G., 1984, Paris, Gallimard (Folio), 2011 (1 ère éd. 1948), expression reprise par Dal Lago A., Non-persone. L'esclusione dei migranti in una società globale, Milan, Feltrinelli, 1999. L'invisibilité absolue traitée ici se situe, donc, en amont de celle, sociale, que subiront les survivants dans les sociétés d'arrivée, $c f$. Le Blanc G., Dedans, dehors. La condition d'étranger, Paris, Seuil, 2010. 
Cependant, sur l'île de Lampedusa, sont parfois visibles des traces mortuaires : on y affronte les morts en mer, les cadavres retirés aux flots, les objets personnels rejetés sur le rivage, le deuil des survivants, les rituels impossibles. Ce fut le cas, notamment, lors du naufrage du 3 octobre 2013, tout près de la côte de l'île, qui fit 150 disparus, 366 morts retrouvés et 148 survivants. La plupart étaient érythréens, certains étaient somaliens ou soudanais, tous fuyaient la violence politique de leur pays. Un second naufrage a eu lieu le 11, entre Lampedusa et Malte, et a fait sans doute 200 disparus, 34 morts retrouvés et 206 survivants, la plupart syriens ${ }^{14}$. Cet événement marque un point de rupture dans la longue liste de naufrages de migrants en Méditerranée. Médiatisé et politisé, il signe l'inscription définitive du thème des naufrages migrants en Méditerranée dans le débat public, même s'il n'entraîne pas, ou peu, de changement de politiques ${ }^{15}$.

Il s'agit ici de comprendre comment cet énième naufrage 16 a pu devenir un point de rupture. Si l'évènement peut être défini comme « une rupture sociale d'intelligibilité 17 », ce naufrage, en introduisant la nécessité d'interpréter l'inconvenance de ces morts visibles, constitue bien une rupture dans la possibilité de donner du sens : il fait surgir de la violence, de l'indécence, de l'émotion. Il rend nécessaire un travail d'interprétation qui va tenter de produire de nouveaux cadrages de la mort aux frontières maritimes de l'UE. Ce travail s'effectue dans les échanges, et les concurrences, entre des groupes d'acteurs sociaux - médias, responsables politiques nationaux et européens, militants - qui traitent l'événement à partir de leurs savoirs collectifs propres : sur ces bases, toutes les prises de position semblent tourner autour de l'enjeu central du degré de visibilité/invisibilité des morts ${ }^{18}$. Pour reconstituer les processus qui contribuent à produire une nouvelle intelligibilité, l'étude minutieuse des différentes interventions publiques autour du naufrage, d'une

14. Les survivants de ce second naufrage ont été transportés soit à Lampedusa soit à Malte ; moins médiatisé, car peu visible, ce naufrage est souvent traité, dans les réactions politiques, en même temps que celui du 3 octobre.

15. On ne s’intéresse ici qu'aux interprétations de l'événement, non aux changements de politiques publiques qu'elles ont, ou non, entraînés. De même, par souci de cohérence, on ne s’intéresse qu'aux morts et disparus de ce naufrage, un autre travail étant nécessaire sur les survivants et sur leur destin, entamé en Europe par un autre scandale moral, celui des mauvais traitements subis au centre d'accueil de Lampedusa, voir Geffroy L. " "Si ce sont des hommes" : la vidéo choc du CPSA de Lampedusa », Courrier international, 18 décembre 2013.

16. Bruno M., «L'ennesimo sbarco di clandestino. La tematica dell'arrivo nella communicazione italiana », in Binoto M., Matino V., Morcellini M. (eds.), Fuori luogo. L'immigrazione e $i$ media italiani, Cosenza, Pellegrini / Rome, Rai, 2003, pp. 95-107.

17. Bensa A., Fassin E., «Les sciences sociales face à l'événement », in "Qu'est-ce qu'un événement ? ", Terrain, 38, 2002, pp. 5-20.

18. L'étude du travail d'interprétation s'appuie sur le concept de " cadre d'interprétation " (frame) de Goffman E., Les cadres de l'expérience, Paris, Minuit, 1991, ainsi que sur l'utilisation de la notion par J. Butler quand elle étudie les réactions à la violence selon les effets de cadrages construits par les médias et par la photographie : Butler J., Ce qui fait une vie. Essai sur la violence, la guerre et le deuil, Paris, Zones, La Découverte, 2010. L'analyse des glissements de sens et des concurrences interprétatives est inspirée de la démarche de sociologie argumentative de Chateauraynaud F., Argumenter dans un champ de forces. Essai de balistique sociologique, Paris, Éditions Petra, 2011. 
part, l'analyse des interprétations des faits qui sont alors énoncées, d'autre part, permettent de suspendre le temps trop rapide de l'événement : on tente ainsi de saisir le jeu des glissements de sens successifs qui ont construit cet événement comme un point d'inflexion du cadrage des naufrages de migrants ${ }^{19}$.

Dans un premier temps, celui d'un fait spectaculaire dont le cadrage initial est donné par les médias, les migrants noyés sont interprétés comme des corps, dont le traitement porte une charge émotionnelle forte. Cette exposition de la mort affiche le drame humain aux yeux de tous 20 et constitue la violence de l'événement (1).

Avec l'entrée en scène des acteurs institutionnels, le cadrage est modifié. Les morts sont interprétés, aussi, comme problèmes de politique publique : qui est en charge du secours ? qui a la responsabilité d'éviter ces naufrages ? qui contrôle la frontière ? Les morts, alors plus « euphémisés » qu'invisibles, deviennent des moyens de pression dans les controverses pour la prise en charge de la responsabilité de la frontière entre échelles de gouvernement, notamment entre l'Italie et l'UE, entre l'Italie et les autres États européens (2).

Cependant, un autre ensemble d'interprétations, militantes, cherchent le sens de ces morts non pas tant à partir de l'événement du naufrage, qu'en fonction d'une vision éthique et politique du droit à la migration. Dans ces versions, les morts, outre qu'ils sont considérés comme la preuve de la violence politique, sont traduits en personnes et en sujets de droits. Des mobilisations tentent alors, pour peser sur les politiques, de donner à voir l'invisible, en multipliant les actions symboliques; elles essaient de rendre visibles les victimes en leur rendant un visage, une histoire, une identité, et en célébrant le deuil (3).

\section{"Ces gens là sont morts, ce ne sont plus des migrants 21 »: Rupture de l'invisibilité à la frontière maritime.}

En Méditerranée, la règle européenne tue : la souveraineté y expose à la mort en autant de « crimes de paix 22 ». Dès l'instauration des visas pour les ressortissants d'un certain nombre de pays extérieurs, dans le cadre de l'application des accords de Schengen, il y eut des tentatives de passage irrégulier par la mer et il y eut des noyés ${ }^{23}$. Malgré les efforts des militants, face à ces

19. Voir la «boîte noire méthodologique » en fin de texte.

20. Cette étape correspond à la « topique du sentiment » telle que l'analyse Boltanski L., La souffrance à distance. Morale humanitaire, médias et politique, Paris, Gallimard (Folio), 2007.

21. "Le travail de Frontex, c'est la lutte contre l'immigration illégale, pas le sauvetage en mer, et ces gens-là sont morts, ce ne sont plus des migrants ", remarque d'un fonctionnaire (anonyme) de Frontex rapportée par Manach J.-M., " Ces gens-là sont morts, ce ne sont plus des migrants », Le Monde diplomatique, 31 mars 2014.

22. Albahari M., 2015, op. cit.

23. Sur la question du comptage des morts en mer, voir l'introduction de ce numéro. 
drames, l'indifférence l'emporta toujours ${ }^{24}$. Bien qu'en la matière les chiffres soient par essence incertains, l'Organisation internationale pour les migrations (OIM) estime qu'en 2011, année des " printemps arabes », au moins 2000 personnes ont disparu en Méditerranée, qu’elles furent plus de 3000 en 2014 et plus de 3760 en 2015 . Le nombre des morts aux frontières de l'UE depuis l'an 2000 est estimé, selon les sources, entre 23000 et 29000 , pour la plus grande partie disparus en Méditerranée ${ }^{25}$. Longtemps, la Méditerranée centrale, en particulier, a englouti des navires fantômes, sans que nul ne s'en émeuve. Ainsi en est-il de la terrifiante histoire des «fantômes de Portopalo », quand périrent 283 Indiens, Pakistanais et Tamuls, durant la période de Noël 1996, dans ce qui était alors le plus grave naufrage en Méditerranée depuis la guerre : les autorités et les médias doutèrent longtemps de la réalité de ce naufrage, qui fut révélée, dans une ambiance de crise morale, par un livre célèbre ${ }^{26}$. Cette révélation reposait sur le témoignage d'un unique témoin local, qui raconta que les pêcheurs du lieu, pour ne pas compromettre leur activité, rejetaient à la mer les cadavres pris dans leurs filets et que le village avait choisi de taire ces incidents. Ainsi en est-il aussi de la disparition de 320 personnes, originaires de l'Afrique sub-saharienne, en avril 2011, après que deux bateaux de pêche aient été obligés à appareiller, sous la menace de militaires libyens, du port de Zuwara vers les côtes italiennes. Un militant révéla ce naufrage ignoré, grâce au seul témoignage d'un demandeur d'asile ${ }^{27}$. Si ces événements remarquables furent quasi invisibles dans l'espace public, que dire des disparitions quotidiennes, que les médias mentionnent comme une litanie depuis les années 1990 : «Hommes avalés par la mer, hommes peut-être arrivés sur nos côtes, mais ensuite disparus, devenus fantômes 28 ?

24. «Les journaux nous parlent des vies perdues et nous en donnent souvent les nombres, mais cela se répète chaque jour et la répétition semble infinie, irrémédiable. Et nous devons ainsi nous demander ce qu'il faudrait non seulement pour appréhender la précarité des vies perdues dans la guerre, mais pour que cette appréhension coincide avec une opposition éthique et politique aux pertes impliquées par la guerre ", Butler J., 2010, op. cit., p. 18. Cette réflexion consacrée aux victimes de la guerre peut être étendue à la situation où les migrants disparaissent victimes de la règle européenne ; on rejoint ainsi la notion de « violence structurelle » aux frontières, exposée par Weber L., Pickering S., Globalization and Borders: Death at the Global Frontier, New York-Basingstoke, Palgrave Macmillan, 2011.

25. Ces données concernent uniquement les morts signalées ; on ignore le nombre réel de disparitions. Il s'agit donc de nombres minimaux et provisoires. Organisation internationale pour les migrations, Fatal Journeys: Tracking Lives Lost during Migration, Genève, 2014 ; Organisation internationale pour les migrations, Mediterranean Update. Missing Migrants Project, Genève, juin 2015 [http://www.iom.int/].

26. Bellu G. M., I fantasmi di Portopalo. Natale 1996 : la morte di 300 clandestini e il silenzio dell'Italia, Milan, Mondadori, 2004. L'auteur est journaliste à La Repubblica et préside l'association Carta di Roma. Voir aussi Ritaine E., « Dramaturgie de l'intrusion migratoire : teatro all'italiana », in Martin D.-C. (ed.), L'identité en jeux. Pouvoirs, identifications, mobilisations, Paris, Karthala, 2010, p. 201-221.

27. À ce moment de la guerre en Libye, le régime de Kadhafi utilisait l'envoi forcé de migrants africains comme une arme de rétorsion contre l'Italie qui abrite des bases de l'OTAN, voir Del Grande G. [http://fortresseurope.blogspot.fr].

28. Nicolini G. (maire de Lampedusa), «Lettera del sindaco alle madri dei migranti tunisini dispersi », juillet 2013 [http://www.storiemigranti.org/spip.php?article1054]. 
A Lampedusa, le 3 octobre 2013, les circonstances sont différentes : le naufrage a eu lieu tout près des côtes, de nombreuses personnes ont participé au sauvetage et témoignent, médias et responsables politiques sont alertés depuis plusieurs années par des mobilisations militantes et par les protestations de la population locale. La nature du naufrage entraîne, cette fois, une couverture médiatique immédiate et mondiale. Sur l'île, envahie par les médias, des traces mortuaires sont visibles, et encombrantes. Les corps des migrants n'ont pas simplement disparu en mer : cette fois-ci, les cadavres sont visibles. Sont évoqués et montrés en boucle, dans les médias ${ }^{29}$, des cadavres (flottant en mer ou échoués sur les plages); des sacs mortuaires; des cercueils ; des objets rejetés par la mer; des dépositoires (les sacs mortuaires, puis les cercueils, alignés dans un entrepôt de l'aéroport ; les cercueils rangés sur le port, puis transportés, par des engins de levage, sur un navire militaire partant vers la Sicile ${ }^{30}$ ); des rescapés en couvertures de survie (surtout des femmes et des enfants), des sauveteurs épuisés équipés de masques de protection, des personnes en pleurs. Les pouvoirs publics sont alors confrontés, d'abord, à la question de la gestion sanitaire de ces corps, dans un contexte îlien démuni. Les corps des noyés sont si nombreux que la place et les moyens manquent, pour les conserver, pour les transporter, pour les enregistrer, pour les inhumer. La maire de Lampedusa revient souvent sur cet aspect : «Venez compter les morts avec nous ", écrit-elle au président du Conseil italien, le lendemain du naufrage; « Nous ne savons plus où mettre les vivants, et les morts non plus »; " J'ai demandé aux sauveteurs de me décrire la situation, ils m’ont répondu que la mer est pleine de morts $31 »$. La gestion des corps suppose, aussi, leur inhumation, or la question du manque de places dans le cimetière est récurrente à Lampedusa ("Quelle taille le cimetière doit-il atteindre sur mon île ? 32 » demande la maire dès 2012, face à d'autres naufrages). Il s'avère donc nécessaire de transporter les cercueils hors de l'île, et de les disperser dans les communes siciliennes qui veulent bien les inhumer ${ }^{33}$. Ce surgissement de la mort, dans ses aspects les plus choquants, induit une première mise en récit, celle d'un drame humain. Ce premier cadrage, en centrant le récit sur l'émotion et sur le spectaculaire, favorise la reprise, par la presse, des théma-

29. Voir par exemple ces photographies sur La Repubblica [http://palermo.repubblica.it/ cronaca/2013/10/03/foto/le_immagini_del_naufragio-67790902/1/?ref=search\#7].

30. Voir par exemple ces photographies : Baster T., Merminod I., "For Eritrean migrants, there is more dignity in death" (Photo Essay), New Internationalist, 18 octobre 2013 [http://newint.org/].

31. Chronique des événements heure par heure [http://cronacaeattualita.blogosfere.it/ post/501313/naufragio-a-lampedusa-] ; voir aussi Jozsef E., "Lampedusa : on n'arrivait pas à séparer les corps pour les remonter », Libération, 17 octobre 2013.

32. Nicolini G., « L'appello del sindaco di Lampedusa all’Unione Europea. Una lettera del nuovo sindaco di Lampedusa », 15 novembre 2012 [http://www.nobordersmagazine.org/2012/ 11/appello-giusi-nicolini-lampedusa-unione-europea/].

33. Il y avait aussi sur l'île un "cimetière de bateaux " (confisqués aux passeurs) dont l'image est devenue emblématique des naufrages de migrants en Méditerranée. Elle a été reprise par les médias du monde entier, sans doute aussi pour sa puissance esthétique. Il est symptomatique de l'action d'euphémisation du drame que ce dépôt ait été détruit par les pouvoirs publics au début de l'année 2015. 
tiques de douleur, de deuil, d'empathie, d'indignation et de honte ${ }^{34}$. L'image, dans ce cas tragique et répétitive, constitue l'événement en diffusant la violence des faits et l'émotion que celle-ci suscite ${ }^{35}$.

Au-delà de la gestion sanitaire apparaît, très vite, le problème de la célébration du deuil : comment rendre hommage aux disparus ? L'organisation d'un rituel de deuil va être très chaotique en Italie, révélant bien que « le deuil public est lié à l'indignation, et (que) l'indignation devant l'injustice ou la perte insupportable a un énorme potentiel politique 36 ». Dans l'émotion initiale est décrété un deuil national, le 4 octobre, lendemain du naufrage, sur proposition du président du Conseil, E. Letta. Le 9, lors de la visite sur place des représentants européens et italiens, E. Letta annonce aussi l'organisation de funérailles d'État, annonce reprise et approuvée par la presse du monde entier. Celles-ci n'auront jamais lieu, cependant. Entretemps, en effet, les cercueils ont été répartis entre de nombreuses communes siciliennes et ne sera finalement organisée qu'une cérémonie symbolique, le 21, à Agrigente, en présence de représentants du gouvernement, mais sans les dépouilles. Cette cérémonie a soulevé de nombreuses polémiques : les survivants, retenus dans le centre de secours de Lampedusa, n'ont pu s'y rendre et en ont été réduits à célébrer leur deuil en défilant dans les rues de l'île 37 ; les habitants de Lampedusa n'ont pas été associés à l'hommage, malgré les protestations de leur maire ; l'ambassadeur d'Erythrée, pays dont la plupart des victimes fuyaient la dictature, a été invité à la cérémonie par le gouvernement italien, ce qui a horrifié les réfugiés ${ }^{38}$. Pour les observateurs, ce rituel de deuil est « une farce, une farce dramatique mise en scène par le gouvernement de ce pays. L'Italie a réussi pour la première fois à organiser des funérailles sans cercueils. Les morts n'étaient pas là (...). Les vivants ne savent pas où les pleurer, ne savent pas qui pleurer (...). Émotion, larmes ministérielles, engagements solennels : tout s'est terminé par la farce d'Agrigente 39 ». En revanche, de petits rituels alternatifs ont eu lieu, loin des cercles nationaux, enracinés dans la piété locale : les communes siciliennes qui ont accepté d'accueillir des

34. Belluati M., «Lampedusa, 3 ottobre 2013. Cronaca di una tragedia », Osservatorio Carta di Roma, Notizie alla deriva, 2014, pp. 63-79 [http://www.cartadiroma.org] ; Bruno M., «Frame e discorsi televisivi nel raconto del dolore. Il naufragio di Lampedusa nei talk italiani ", Notizie alla deriva, op. cit., pp. 80-98. Voir aussi le schéma des co-occurrences textuelles avec le terme Lampedusa dans la presse écrite (Belluati M.) et le tableau des titres de talk shows italiens consacrés au naufrage (Bruno M.) publiés sur [http://enigmur.hypotheses.org/2683].

35. Sur l'analyse de l'émotion face aux événements violents, voir les remarques de M. Delori dans Bertrand G., Delori M., Terrorisme, émotions et relations internationales, Bordeaux, Myriapode, 2015.

36. Butler J., 2010, op. cit., p.43.

37. Messina P., « Lampedusa, l'altro funerale dei sopravvisuti », L’Espresso, 21 octobre 2013.

38. Bellu G. M., "Strage di Lampedusa, la denuncia di Alganesh : "I politici sono peggio degli scafisti” », 9 septembre 2014 [http://notizie.tiscali.it/articoli/cronaca/14/09/09/ bellu-migranti.html].

39. Fierro E., «Agrigento, la farsa dei funerali alle vittime di Lampedusa. Senza morti né vivi », Il Fatto quotidiano, 21 octobre 2013 ; voir aussi AFP, «Lampedusa : cérémonie commémorative sans les survivants du naufrage », lapresse.ca, 21 octobre 2013. 
dépouilles dans leur cimetière ont procédé à des commémorations interconfessionnelles et Lampedusa a inventé ses propres rituels (messes, processions, commémorations interconfessionnelles). La difficulté à assumer le deuil culmine, enfin, avec la longue absence d'une procédure d'identification des corps : ceux-ci restent une foule anonyme et effaçable. La plupart ont été inhumés sous un numéro matricule correspondant à une description sommaire et la moitié demeurent non identifiés. Ce n'est qu'en août 2014, soit presqu'une année plus tard, qu'est enfin mise en place une procédure d'identification pour les familles de victimes, "car il n'y a pas de morts de série B 40 ».

Ces morts de Lampedusa ne sont donc pas radicalement invisibles, comme le sont les morts fantômes de la Méditerranée : car la Méditerranée n'est pas tant, comme on l'écrit souvent, un cimetière (institution qui suppose des rituels de deuil) qu'une fosse commune où s'engloutissent des vies qui ne peuvent être pleurées, "une généralité indifférenciée " selon les mots d'A. Mbembe ${ }^{41}$. Cependant, les morts de Lampedusa demeurent pour la plupart inconnus, et non célébrés. Ils sont soit des nombres, soit des cadavres anonymes. Un journaliste qui a couvert l'événement écrit ainsi : «"Mort numéro 31, sexe masculin, noir, probablement trente ans"; "Mort numéro 54, sexe féminin, noire, probablement vingt ans"; "Mort numéro 11, sexe masculin, noir, probablement trois ans". Probablement : c'est tout ce que nous savons d'eux. Dans ce mot, il y a toute leur vie. Quand ils sont morts, ils sont morts, c'est ainsi que nous les racontons : probablement ${ }^{42}$ ».

Le ratage des rituels de deuil (ils ne peuvent être pleurés) est le signe du non-être politique de ces morts : ils sont comme tombés dans une faille du politique. La visibilité même relative de leur disparition, attestée par les images mortuaires mais « euphémisée » par l'anonymat de leur sépulture, fait scandale et appelle un traitement politique : il n'est pas possible, cette fois, de simplement regretter de fâcheuses statistiques et de s'en tenir à des généralités. Le cadrage de l'événement par les médias est donc rapidement modifié par l'apparition des acteurs institutionnels. Ceux-ci entreprennent, dans le même mouvement, de participer au rituel de déploration et de traduire l'événement en termes (de) politiques.

40. Ciolli B., "Strage di migranti, Cristina Cattaneo : "Non esistono morti di serie B" ", lettera43, 12 avril 2014. A propos du Laboratorio di antropologia e odontologia forense (Labanof) dell'Istituto di Medicina legale della Statale di Milano [http://www.lettera43.it/].

41. Mbembe A., op. cit., p. 53.

42. Bolzoni A., "Cosà scivero dopo quell'immagine ?", Notizie alla deriva, op. cit., p. 52. L'auteur est journaliste à La Repubblica. Traduction du titre : "Qu'écrirai-je après cette image?» 


\section{«Plus jamais cela » : L’invisibilité entretenue par les routines politiques}

Les responsables politiques en charge de la gestion de la frontière, membres du gouvernement italien et représentants de la Commission européenne, interviennent pour coder l'événement selon leurs propres « contextes d'interprétation $43 »$ : registre de l'émotion et registre du politique se conjuguent alors en des accords changeants. Les opérations d'interprétation des acteurs institutionnels s'effectuent sur deux lignes complémentaires : la déploration et l'annonce de mesures à forte charge symbolique; la mise en concurrence de propositions politiques et l'apparition de controverses. Ces acteurs déploient ainsi l'habituelle thématique de l'urgence (en italien, emergenza) qui, en matière d'immigration, est paradoxalement devenue un argument routinier. Elle permet d'attester d'une situation de crise et d'en appeler au partage des responsabilités avec les autres instances politiques, nationales comme européennes : les morts deviennent alors des problèmes de politiques publiques.

La séquence de la déploration commence dès les premières heures et se déroule la plupart du temps sur les lieux mêmes ; elle est marquée par la tonalité émotionnelle des déclarations. Selon un rituel public établi, elle obéit à la logique des positions institutionnelles. G. Napolitano, le président de la République, qui, en Italie, est une autorité morale, parle d'un « massacre des innocents 44 ». Le ministre de l'Intérieur et vice-président du Conseil, A. Alfano 45 est le premier sur place, le 4 octobre : « (J'ai vu) une scène effroyable que je n'aurais jamais imaginé voir. Une scène qui offense l'Occident, l'Europe ». Devant cette «immense tragédie », le président du Conseil, E. Letta, annonce une journée de deuil national ${ }^{46}$. La présidente de la Chambre des députés, L. Boldrini, arrive sur les lieux le 5: «Tout le pays étreint l'île et les familles des morts et des disparus de cette épouvantable tragédie 47 ». Puis la ministre de l'Intégration, C. Kyenge, elle-même d'origine immigrée et souvent attaquée en tant que telle, vient se recueillir sur les lieux, le 7 : «Sur cette barque, à la place de ces désespérés, cela aurait pu être moi (...).

43. Bensa A., Fassin E., op. cit.

44. [http://www.ilfattoquotidiano.it/2013/10/03/naufragio-lampedusa-lega-attacca-boldrini-ekyenge-colpa-loro/731387/]

45. Ministre de l'Intérieur au moment du naufrage de 2013 (gouvernement Letta), (à nouveau) ministre de l’Intérieur dans le gouvernement Renzi, installé en février 2014. Auparavant, il a été en 2008 ministre de la Justice du gouvernement de S. Berlusconi et était présenté comme son «dauphin "; en 2011, il est devenu secrétaire général du PDL (Popolo della Libertà / Forza Italia), parti berlusconien; il a rompu avec celui-ci en novembre 2013 et fondé avec une cinquantaine de députés le Nuovo Centro Destra (NCD). Sources successives pour ses déclarations : Compte Twitter personnel; Communication urgente à la Camera dei deputati, 4 octobre 2013 [http://www.angelinoalfano.it/] ; Émission télévisuelle « La telefonata di Belpietro », Canale 5, 4 octobre 2013, en direct de Lampedusa.

46. E. Letta est un homme politique chrétien social, membre de La Margherita, puis de l'Ulivo, puis du Partito Democratico (centre gauche réformiste), plusieurs fois député et ministre, président du Conseil des ministres italien d'avril 2013 à février 2014.

47. Communiqué de presse, 4 octobre 2013 ; L. Boldrini est journaliste, ancienne porte-parole du Haut Commissariat des Nations unies pour les réfugiés, élue à la Chambre des députés sur une liste Sinistra, Ecologia, Libertà (SEL). 
Ces morts, nous les avons tous sur la conscience 48 ». Le rituel de déploration se poursuit lors de la visite des responsables européens sur l'île, le 9 octobre. J.M. Durao Barroso (président de la Commission européenne) déclare : "C'est une image qui ne peut s'oublier, il y avait des cercueils de petits enfants, de mères et d'enfants ». C. Malmström (Commissaire européenne aux affaires intérieures) parle «d'une immense douleur devant les cercueils à Lampedusa » et déclare que « ce n'est pas digne de l'Europe ». Cependant que le président du Conseil italien renouvelle « ses excuses pour les défaillances de (son) pays face à une telle tragédie » et annonce des funérailles nationales ${ }^{49}$.

La réserve d'un temps de recueillement n'existe pas, cependant : la déploration n'est jamais exempte de proposition politique. Les déclarations des premières heures, elles-mêmes, n'échappent pas à cette tendance. Le président de la République, lorsqu'il évoque, le 3, un " massacre des innocents », enchaîne en appelant à lutter contre le «trafic criminel d'êtres humains » et à renforcer l'action européenne grâce à Frontex ${ }^{50}$. Dès le 3, le ministre de l'Intérieur propose un ensemble de mesures : "Nous ferons entendre haut et fort la voix de l'Italie en Europe pour modifier l'accord de Dublin qui fait trop peser la charge sur les pays d'entrée (...). Il faut parvenir à un système de protection des frontières, cette mer ne marque pas la frontière entre l'Afrique et Lampedusa, mais entre l'Afrique et l'Europe, la protection doit être européenne. Les trafiquants de mort doivent être arrêtés par une action internationale et européenne 51 ». Le 4, devant la Chambre des députés, présentant son programme pour le Conseil européen Justice et Affaires intérieures qui doit se tenir quelques jours plus tard, il précise: « (À l'avenir) pour l'Europe protéger ses propres frontières signifiera protéger ses propres citoyens, mais aussi protéger de la mort ceux qui franchissent sans protection ces frontières, aux mains de marchands de mort (...). À Luxembourg, la semaine prochaine, l'Italie demandera que soit dépassée l'approche si bureaucratique du Règlement de Dublin, qui fait peser la charge des migrants sur le pays d'entrée 52 ». Les morts, corps et identités de personnes, sont ainsi immédiatement traduits dans une version généralisante, celle du langage de l'action : protéger de la mort, réprimer le trafic d'êtres humains, condamner les marchands de mort, gérer les survivants. Ces catégories sont destinées à devenir des ressources dans les controverses politiques sur la prise en charge des migrants, morts ou vifs : les morts entrent ainsi dans les corridors des institutions.

48. Déclaration faite le 4 octobre, La Repubblica, 4 octobre 2013 ; C. Kyenge, médecin, membre du Partito Democratico, est ministre de l'Intégration du gouvernement Letta, 2013-2014. Elle est la première femme d'origine africaine à devenir ministre en Italie, $c f$. Ridet $\mathrm{P}$., " Cécile Kyenge, l'“étrangère" du gouvernement italien ", Le Monde, 4 juillet 2013.

49. Pour ces trois déclarations [http://tg24.sky.it/tg24/cronaca/2013/10/09/lampedusa proteste_alfano_letta_barroso.html].

50. Op. cit., note 44 ; Frontex : Agence européenne pour la gestion de la coopération opérationnelle aux frontières extérieures.

51. «La telefonata di Belpietro ", Canale 5, 4 octobre 2013, op. cit.

52. [http://www.angelinoalfano.it/s6-attivita/17000/angelino-alfano-europa-protegga-propriafrontiera/] 
En effet, à la fin du même mois d'octobre, les 24 et 25 , doit se réunir à Bruxelles un Conseil européen durant lequel l'Italie veut obtenir la solidarité européenne pour la gestion de la frontière. Les morts deviennent alors un moyen de pression pour que les autres États européens acceptent, enfin, de partager la charge du contrôle de la frontière européenne en Méditerranée : ils se font ressources dans la négociation routinière entre les États méridionaux et les autres ${ }^{53}$. Le gouvernement italien tente d'imposer à ses partenaires, au travers du cadrage de l'urgence et de l'obligation morale du sauvetage, un renforcement communautaire des moyens de contrôle de la frontière maritime. Pour cela, à la mi-octobre, il décide de lancer une opération de surveillance et de secours, basée sur ses propres moyens militaires, et nommée Mare Nostrum ${ }^{54}$. Cette opération n'est qu'une augmentation de moyens pour la surveillance et le secours en Méditerranée centrale que les forces armées italiennes effectuent depuis le début des années 1990 : comme toujours dans le contrôle de la frontière, il s'agit d'une opération où sécuritaire et humanitaire s'entremêlent ${ }^{55}$. Mais, en accentuant discursivement sa vocation de secours ${ }^{56}$, le gouvernement italien se forge des ressources argumentatives face aux autorités européennes et aux autres États européens, rétifs à la communautarisation de la gestion de la frontière. En effet, le président du Conseil la présente ainsi : "Pour nous il est intolérable que la Méditerranée soit une mer de mort (...). Nous sommes face à un changement d'époque en matière d'immigration et cela impose un changement radical des normes et de l'action aux niveaux européen et national. Mais nous ne pouvons pas attendre selon les rythmes des institutions et du Parlement européen, quand sont en cause des vies humaines. Nous devons agir immédiatement et c'est pourquoi le gouvernement à décidé de lancer une mission humanitaire ». Le ministre de la Défense, M. Mauro, lui, précise bien que « ce sera une opération militaire et humanitaire, qui renforcera le dispositif de surveillance et de secours en haute mer pour augmenter le niveau de sécurité des vies humaines (...). (Il s'agit de) faire comprendre clairement à l'Europe qu'il n'est pas question pour l'Italie de se désengager, mais qu'il faut aussi que ses partenaires s'engagent 57 ». Le 22, E. Letta, présentant à

53. Ritaine E., «La fabrique politique d'une frontière européenne en Méditerranée. Le “jeu du mistigri" entre les États et l'Union », Les Études du CERI, 186, 2012.

54. «Sangue Nostrum» répondent certaines banderoles lors de manifestations de défense des immigrés en Italie.

55. Cuttitta P., "From the Cap Anamur to Mare Nostrum. Humanitarianism and Migration Controls at the EU's Maritime Borders", CLEER Working Papers, 2014, 7, pp. 21-37; Tazzioli M., "The Desultory politics of Mobility and the Humanitarian-Military Border in the Mediterranean. Mare Nostrum beyond the sea”, REMHU-Revista Interdisciplinar da Mobilidade Humana, XXIII, 44, 2015, pp. 61-82.

56. La réalité du secours est par ailleurs indéniable puisque environ 155000 personnes ont été sauvées en 2014 durant l'opération Mare Nostrum, selon le ministre de l'Intérieur italien G. Pinto, Cuttitta P., 2014, CLEER, op. cit., p. 26.

57. «Immigrazione, al via operazione Mare nostrum », La Repubblica, 14 octobre 2013. Signe de la dominante militaire de l'opération, à la conférence de presse faisant suite à la réunion interministérielle sur l'urgence immigration à Lampedusa, durant laquelle est présentée l'opération Mare Nostrum, sont présents le ministre de l'Intérieur et vice-président du Conseil A. Alfano et le ministre de la Défense M. Mauro ; les forces armées sont représentées par le Chef d'état-major de la Défense (Amiral L. Binelli Mantelli), et le Commandant général de la 
la Chambre des députés ses objectifs pour le Conseil européen, précise parfaitement la pression italienne : « Dans la salle du Conseil, entreront pour la première fois la douleur, la mort, la frustration et la honte que la tragédie de Lampedusa a provoqué et continue de provoquer (...). L'Europe, par son histoire, par ses plus profonds principes fondateurs, ne peut rester à regarder : si elle le fait, l'Europe meurt. Elle meurt avec les centaines d'hommes, de femmes et d'enfants qui perdent la vie en cherchant le salut 58 ».

En fait, malgré ce déploiement d'arguments éthiques, les routines institutionnelles l'ont emporté. Le Conseil européen d'octobre décide de ne rien décider avant les élections européennes de juin 2014. Le semestre de présidence de l'UE par l'Italie, en 2014, qui, sous l'égide du gouvernement M. Renzi, devait faire de la question un enjeu central pour l'UE, échoue également. Aussi, le gouvernement italien suspend-il l'opération Mare Nostrum au bout d'une année, en octobre 2014, en arguant de son coût excessif pour la seule Italie : modeste résultat de la négociation, elle est remplacée par une opération de surveillance de Frontex, Triton, aux moyens bien plus limités. L'événement du naufrage à Lampedusa semble ainsi n'avoir été, sur le plan politique, qu'une bifurcation imparfaite ${ }^{59}$. Certes, l'interprétation politique du naufrage de Lampedusa a contribué à fonder la légitimité de « la scène du secours 60 » en Méditerranée : il n'est plus possible de ne pas secourir, ou de mal secourir. Cependant, la thématique de l'urgence, traduite en obligation de sauvetage et nécessité de lutte contre les trafiquants, a permis d'éviter de poser la question cruciale d'un changement de politique européenne en matière d'immigration et d'asile : elle n'a pas permis l'émergence d'une remise en cause du coût humain du contrôle de la frontière, du cercle vicieux où les mesures sécuritaires elles-mêmes induisent un risque mortel pour les migrants et rendent donc nécessaires les mesures humanitaires.

Dès les premiers mois de 2014, les naufrages ont repris, inéluctables, avec leur cortège de nouveaux morts anonymes. Des disparus d'octobre 2013, il n'est plus guère question au bout de quelques mois : «Ce n'est pas seulement qu'un "discours" déshumanisant produit ces effets, mais plutôt que la limite du discours établit la limite de l'intelligibilité humaine. Ce n'est pas seulement qu'une mort est à peine remarquée, mais qu'elle ne peut l'être. Une telle mort disparait, non dans le discours explicite, mais dans les ellipses par lesquelles

Capitainerie du port (Amiral F. Angrisano) [http://www.dailymotion.com/video/ x15yzv8_roma-presentazione-dell-operazione-mare-nostrum-14-10-13_news].

58. [http://www.enricoletta.it/primo-piano/dinanzi-a-quel-che-succede-a-lampedusa-]

59. Les bifurcations sont définies comme des séquences d'action, très imprévisibles, qui déterminent, de façon irréversible, de nouvelles orientations d'action, Grossetti M., Sociologie de l'imprévisible. Dynamiques de l'activité et des formes sociales, Paris, PUF, 2004 ; Bessin M., Bidart C., Grossetti M. (eds.), Bifurcations. Les sciences sociales face aux ruptures et à l'événement, Paris, La Découverte, 2010.

60. Tazzioli M., "The Politics of Counting and the Scene of Rescue. Border Deaths in the Mediterranean”, Radical Philosophy, 192, 2015 [http://www.radicalphilosophy.com]. 
procède le discours public 61 ». Aussi, les protestations les plus véhémentes contre ce naufrage ne peuvent-elles que s'appuyer sur la volonté de rendre visibles ceux qui ont disparu, et doublement disparu dans les ellipses des discours publics. Si celles-ci brouillent la visibilité des morts, alors des interprétations protestataires s'attachent à dévoiler ce qui se cache derrière ces morts : il faut les sortir de l'espace de la "vie nue ", "au travers d'actes politiques de remémoration, de récupération et de reconstruction, (qui font) retourner les corps vers le présent, où ils font sens en relation à d'autres corps, vivants et morts 62 ».

\section{Contre « la globalisation de l'indifférence 63 : Les luttes pour rendre visi- bles les invisibles}

Ceux qui veulent rendre leur visibilité aux disparus font partie d'une " coalition de cause 64 » agissant sur le long terme pour modifier le cadrage de l'immigration irrégulière et les politiques qui en découlent. Soit directement en appelant à un changement de la politique européenne, soit indirectement en cherchant à susciter un sursaut citoyen, ces acteurs défendent les droits humains des migrants en leur redonnant une visibilité humaine : une trace, un visage, une histoire, une identité. Ils trouvent leur inspiration dans l'universalisme chrétien, basé sur la valeur de la personne humaine, emmené par le dynamisme du pape François et appuyé sur les réseaux catholiques italiens; ils sont aussi ancrés dans l'universalisme laïque, celui des droits humains, qui irrigue les prises de position de nombreuses associations militantes et d'intellectuels. Leurs interprétations sont plus indépendantes que les autres de l'événement lui-même. Elles se différencient du cadrage spectaculaire par les médias, car elles sont actives avant, et aussi longtemps après, l'événement. Elles sont décalées des cadrages institutionnels, dont l'horizon est l'action de gouvernement, car elles se veulent expression de la défense des droits humains : elles s'appuient sur ce socle de valeurs pour dénoncer les politiques existantes et pour réclamer des droits pour les migrants.

Aussi ces interprétations se situent-elles dans une temporalité différente : les engagements qui les portent sont présents sur le thème des morts aux fron-

61. Butler J., 2005, op. cit., p.62.

62. Perera S., op. cit., p. 650. L'auteur retravaille la notion de témoignage telle qu'elle est traitée dans Agamben G., Ce qui reste d'Auschwitz, Paris, Rivages, 2003.

63. Homélie du pape François, 8 juillet 2013, Lampedusa [http://w2.vatican.va/content/ francesco/fr/homilies/2013/documents/papa-francesco_20130708_omelia-lampedusa.html].

64. Sabatier P., “The Advocacy Coalition Framework: Revisions and Relevance for Europe”, Journal of European Public Policy, 5/1, 1998, pp. 98-130. La coalition de cause est définie comme un ensemble "d'acteurs issus d'organisations gouvernementales et privées variées, qui, dans le même temps, (a) partagent un ensemble de croyances normatives et causales et (b) participent, dans un degré non négligeable, à une activité coordonnée dans le temps. L'ACF démontre explicitement que la plupart des coalitions n'incluront pas seulement des leaders de groupes d'intérêt, mais également des fonctionnaires d'agences publiques, des législateurs aux différents niveaux de gouvernement, des experts, et peut-être même quelques journalistes » (p. 103). 
tières depuis de longues années (à ce titre, ils agissent comme des lanceurs d'alerte vis-à-vis des médias et des responsables politiques) et ils demeurent actifs après le drame de Lampedusa pour que le souvenir des victimes du naufrage ne s'efface pas (en ce sens, ils sont autant de veilleurs de mémoire).

\section{Les lanceurs d'alerte}

Les lanceurs d'alerte sont des acteurs décisifs car leur engagement sur la longue durée a contribué à sensibiliser les médias et les opinions publiques à la question des naufrages en Méditerranée : c'est aussi grâce à ce travail de sensibilisation que le naufrage de 2013 a été autant médiatisé. Ils assurent, en outre, une pression politique constante car, actifs sur ces thèmes bien avant l'événement du naufrage, ils le demeurent aussi après, forts de leur expertise. Les associations de défense des droits des migrants sont présentes des deux côtés de la Méditerranée depuis de nombreuses années, mobilisées contre la répression lors des franchissements de frontière et contre l'indignité des centres de rétention et des camps informels. Ce sont des réseaux d'organisations européennes et africaines, travaillant en coalitions changeantes, et de plus en plus présents sur les lieux mêmes, comme une expression activiste de la société civile. Peu à peu, la thématique des noyades en Méditerranée s'est imposée à eux. L'opération Boats4People (B4P), organisée en 2011 par un réseau d'ONG euro-africaines, à la suite des nombreuses disparitions en mer après les flux de départs qui ont suivi les printemps arabes et la guerre en Libye, en est l'illustration : elle est lancée comme " une flottille de la solidarité en Méditerranée, (pour) dénoncer l'hécatombe qui a lieu actuellement dans les eaux entre Tunisie et Italie 65 ». Le bateau de B4P a navigué en Méditerranée durant l'été 2012 : son parcours a été l'occasion d'une riche couverture médiatique de la question des migrants en Méditerranée ${ }^{66}$, qui a contribué à amorcer un débat public, bien avant le naufrage de l’automne 2013 .

Depuis plusieurs années occupés à dénoncer le risque mortel encouru par les migrants dans la traversée irrégulière de la Méditerranée, ces réseaux activistes ont été amenés, en désespoir de cause, à prendre aussi des initiatives « urgentistes », en devenant eux-mêmes acteurs de la « scène du secours » : il s'agit d'une veille humanitaire de substitution, mais aussi d'un moyen de braquer les projecteurs sur les responsabilités européennes. L'initiative WatchTheMed, lancée en 2012, " pour exercer un droit de regard en mer et tenir les États pour responsables des morts de migrants et de la violation de leurs droits ", est ainsi une façon de surveiller les surveillants ${ }^{67}$. Le sauvetage

65. [http://www.boats4people.org]

66. Voir, par exemple, la série d'articles de Fouteau C., «La Méditerranée cimetière migratoire », Médiapart, juillet 2012 [http://www.mediapart.fr/dossier/international/la-mediterraneecimetiere-migratoire] ; voir la revue de presse complète de B4P sur [http://enigmur. hypotheses.org/1428].

67. Il s'agit d'une veille en ligne sur les risques de naufrages et les atteintes aux droits des migrants aux frontières maritimes : ce réseau surveille et cartographie quasi en temps réel les cas de 
d'initiative privée s'est beaucoup développé après le naufrage de Lampedusa, comme un reproche éthique face à l'anomie publique. En 2014, un couple d'entrepreneurs vivant à Malte a fondé la Migrant Offshore Aid Station (MOAS) : en partenariat avec Médecins sans frontières (MSF), ils patrouillent avec leur propre navire jusqu'aux abords des côtes libyennes ${ }^{68}$. Une autre initiative privée, Sea-Watch, a été lancée en 2015, par un entrepreneur allemand, et porte secours avec un navire basé à Lampedusa ${ }^{69}$. MSF a également affrété deux bateaux, en 2015, pour la recherche et le secours de naufragés ${ }^{70}$. Cette préoccupation du secours et du respect du droit s'appuie sur une interprétation qui active, en amont, le principe de solidarité avec les migrants et l'existence de droits des migrants, comme le fait le réseau Migreurop : « La politique d'inhospitalité des États européens atteint un niveau tel qu'il est de notre devoir d'agir afin de montrer qu'un espace euro-méditerranéen solidaire et respectueux des droits humains est possible ${ }^{71}$ ».

De nombreux intellectuels jouent aussi les lanceurs d'alerte. Le journaliste G. Del Grande, pionnier du recensement des morts en Méditerranée avec son blog Fortress Europe, fait des reportages depuis de longues années autour du bassin méditerranéen et relève les noms des disparus : il pense que le nombre de morts est « digne d'une guerre 72 ». F. Gatti, journaliste de l'hebdomadaire L'Espresso, voyageur anonyme parmi les migrants d'Afrique sub-saharienne et naufragé volontaire à Lampedusa en 2005 (épopée dont il a tiré un livre ${ }^{73}$ ), enquête sans cesse sur ces thèmes et dénonce les scandales liés à l'immigration. L'écrivain italien E. De Luca est très présent, et depuis longtemps, dans ces protestations : en 2009, depuis Lampedusa, il raconte à la télévision les migrations contemporaines, en rappelant aux Italiens leurs propres émigrations ${ }^{74}$; il consacre, surtout, une de ces œuvres (publiée en 2005) à donner la parole à ceux qui sont partis pour un « aller simple ${ }^{75}$ »:
«Des mains m’ont saisi, douaniers du Nord, gants en plastique et masque sur la bouche.
Ils séparent les morts des vivants, voici la récolte de la mer, mille de nous enfermés dans un endroit pour cent »

détresse en mer ; il a aussi créé, en 2015, un numéro téléphonique d'alerte pour les migrants en mer, qui permet de contrôler l'effectivité des secours, une fois l'alarme donnée [http://www.watchthemed.net/].

68. [http://www.moas.eu/]

69. [http://sea-watch.org]

70. [http://www.msf.org/topics/mediterranean-migration]

71. Communiqué Migreurop, 7 juillet 2011 [http://www.migreurop.org/].

72. [http://fortresseurope.blogspot.com] ; voir aussi Del Grande G., Il mare di mezzo. Al tempo dei respingimenti, Modène, Infinito, 2010.

73. Gatti F., Bilal sur la route des clandestins, Paris, Liana Levi, 2008 (éd. italienne 2007).

74. 20 mai 2009, émission "Che tempo che fa », Rai3 [https://www.youtube.com/ watch?v=gPEmvU3R_i0] ; [http://www.libreidee.org/2010/03/mi4granti-erri-de-luca-noicarcerieri-di-viaggiatori/].

75. De Luca E., Aller simple, Paris, Gallimard, 2012, p. 51 (éd. italienne, Solo andata, Milan, Feltrinelli, 2005). 
Lorsque le naufrage de Lampedusa a lieu, ces réseaux réagissent d'autant plus vivement, que le scandale des morts qu'ils dénoncent depuis longtemps est, cette fois, visible. Dans leur interprétation, il est question d'une guerre aux migrants et d'un assassinat perpétré par l'Europe : «La guerre ? Comment nommer autrement la mise en place délibérée de dispositifs de contrôles frontaliers destinés, au nom de la lutte contre l'immigration irrégulière, à repousser celles et ceux que chassent de chez eux la misère et les persécutions ? (...) Les morts de Lampedusa, comme ceux d'hier et de demain, sont les victimes d'une Europe enfermée jusqu'à l'aveuglement dans une logique sécuritaire, qui a renoncé aux valeurs qu'elle prétend défendre. Une Europe assassine ${ }^{76}$ ». Cette radicalité s'exprime également dans les suites données à l'événement de Lampedusa par le réseau Progetto Melting Pot Europa, lorsqu'il propose, début 2014, la rédaction d'une déclaration des droits des migrants, élaborée par le rassemblement des activistes à Lampedusa : « la charte de Lampedusa, pour opposer (à l'appauvrissement radical des institutions démocratiques) un autre droit, écrit à partir du bas ». La charte de Lampedusa prend, de fait, la forme d'une déclaration des droits, et affirme, pour tous, la liberté de circulation, d'installation, de choix existentiels 77.

Dans ce contexte de mobilisation de longue durée, intervient aussi un acteur local, la maire de Lampedusa, G. Nicolini, qui s'exprime sur ce thème dès son élection, en 2012, et depuis interpelle sans cesse les pouvoirs publics ${ }^{78}$. Elle est devenue une intervenante majeure dans le débat politico-médiatique, par la portée de ses interventions, qui prennent des formes particulières : désespérée de recevoir toujours plus de cadavres sur ses côtes, elle adresse, à peine élue, un appel à l'UE, sous forme de lettre ouverte, en novembre 2012 ; en juillet 2013, elle répond, par une lettre ouverte, aux suppliques des mères de migrants tunisiens disparus. Elle aussi parle d'une guerre, elle aussi est en lutte pour faire reconnaître ces personnes disparues qu'elle adopte symboliquement dans ses discours : « Je suis indignée de l'accoutumance qui semble avoir gagné tout le monde, je suis scandalisée du silence de l'Europe qui vient de recevoir le prix Nobel de la paix et qui se tait face à une tragédie dont les victimes sont celles d'une véritable guerre. (...) Alors, si ces morts sont seulement les nôtres, alors, moi je veux recevoir des télégrammes de condoléances chaque fois qu'un noyé m'est remis. Comme s'il avait la peau blanche, comme si c'était un de nos enfants noyé durant les vacances 79 ». À rebours de la

76. Communiqué du réseau Migreurop, 4 octobre 2013 [http://www.migreurop.org/].

77. [http://www.meltingpot.org/+-Carta-di-Lampedusa-+.html]. Voir le texte final de la charte sur http://enigmur.hypotheses.org/2683

78. Membre de l'association environnementale LegAmbiante, maire depuis mai 2012, élue sur une liste Lista Civica, qui a vaincu de justesse l'équipe sortante précédente, conservatrice, qui avait pris des positions xénophobes lors des débarquements de migrants tunisiens en 2011.

79. "L'appello del sindaco di Lampedusa all'Unione Europea », novembre 2012, op. cit. ; après le naufrage d'octobre, elle affirme encore cette forme d'adoption des morts, avec une photographie des sacs mortuaires publiée sur son compte Twitter : «Questi sono i nostri morti. I primi 93 \#Lampedusa » (trad. : "Ceux-ci sont nos morts, les 93 premiers ») [https://twitter.com/giusi_nicolini/status/385789454310330368/photo/1]. 
logique du contrôle, elle déclare : « Nous, nous espérons qu'il y ait des débarquements, que ces personnes parviennent à accoster, qu'elles arrivent vivantes 80 ». Elle va jusqu'à parler d'un « holocauste moderne » en Méditerranée : pour elle, désormais, " (à Lampedusa en 2013) être parvenu à aligner ces cercueils et à les montrer a supprimé tout alibi 81 ».

Cependant, c'est d'un acteur éminemment global, le pape François, qu'est venue l'alerte décisive. Il a, en effet, pris la décision d'effectuer son premier voyage apostolique à Lampedusa, en juillet 2013, plusieurs mois avant le naufrage, pour honorer les «immigrés morts en mer, dans ces bateaux qui au lieu d'être un chemin d'espérance ont été un chemin de mort ». Ce signal très fort a concentré l'attention médiatique sur l'île, dès le mois de juillet, et fortement mobilisé les cercles catholiques, en Italie et au-delà. Dans son homélie sur place, il condamne l'indifférence et réincarne les disparus : « Dans ce monde de la globalisation, nous sommes tombés dans la globalisation de l'indifférence (...). Nous sommes une société qui a oublié l'expérience des pleurs, du "souffrir avec" : la globalisation de l'indifférence nous a ôté la capacité de pleurer ! 82 ». L'expression " globalisation de l'indifférence » a fait le tour du monde et est devenue celle qui évoque le mieux les drames en Méditerranée. Quand s'est produit le naufrage du 3 octobre, elle est revenue dans tous les titres de presse, elle a été rappelée dans les églises et relayée par les instances catholiques. Ainsi, par exemple, les évêques siciliens considèrent-ils en octobre 2013, en reprenant les paroles pontificales, que la politique est « coupable de ces morts » : "Ces morts, et tous ceux qui durant des années ont disparu en mer, demandent vérité, justice et solidarité 83 ».

\section{Les veilleurs de mémoire}

Alliés objectifs des précédents, les veilleurs de mémoire sont parfois présents sur ce thème par profession, comme les journalistes et les photographes; ils peuvent aussi réagir par une révolte éthique, comme les artistes ou certains habitants de l'île. Pour eux, l'impératif est que ces morts ne soient pas oubliés, dans le tragique de l'événement du naufrage, mais aussi dans l'humanité de leur existence.

Les témoins de profession, journalistes et photographes, sont au premier rang de cette veille : pour eux, il s'agit de donner à voir, au sens fort du terme. L'hebdomadaire L'Espresso est caractéristique de cette prise de position. Son directeur écrit ainsi, en octobre 2013, un éditorial intitulé « Maintenant,

80. Entretien avec AdnKronos, 20 août 2012, cité par Dalla Chiesa N., «Lampedusa, la sindaco gentile che accoglie l'umanità », Il Fatto quotidiano, 10 septembre 2012

81. Fouteau C., "La maire de Lampedusa dénonce "un holocauste moderne" en Méditerranée ", Médiapart, 30 décembre 2013.

82. Homélie du pape François, 8 juillet 2013, Lampedusa, op. cit. À la suite du naufrage du 3 octobre, il s'exprime d'un seul mot : «C'est une honte".

83. Conférence épiscopale régionale, Sicile, La Repubblica-Palermo, 14 octobre 2013. 
cependant, ne pas les oublier 84 », dans lequel il justifie par l'impératif de mémoire la forte récurrence du thème des naufrages dans son magazine. Cette politique éditoriale se maintient au fil des semaines, avec de très nombreux dossiers et couvertures dédiés à Lampedusa et à la question migratoire en général. F. Gatti, l'un des journalistes de l'hebdomadaire, s'est ainsi consacré à retrouver les familles des noyés syriens du naufrage du 11 octobre (second naufrage d'octobre 2013, entre Lampedusa et Malte) et à rassembler les photographies des disparus. En un document choc, il a publié ces photographies sur son blog (juxtaposées à celles des acteurs politiques européens, considérés comme responsables), accompagnées de leur histoire personnelle, sous le titre «Les enfants que l'Europe a donné en nourriture aux poissons », puis en couverture de l'hebdomadaire sous le titre «Laissés mourir 85 ». La ligne éditoriale de La Repubblica est comparable. Le quotidien entretient de riches dossiers sur la question migratoire et se préoccupe de donner à voir ce qui est rarement montré. Ainsi diffuse-t-il, sur son site, les vidéos des différentes opérations de secours, les très dures images tournées par les plongeurs dans l'épave au large de Lampedusa, où les cadavres sont visibles, et surtout l'image d'un couple noyé, enlacé dans la mort, « cette étreinte incroyable dans une mer de mort 86 ».

La mémoire est aussi entretenue, sur l'île, par de nombreux actes symboliques de remémoration. Le plus emblématique est l'installation à la pointe de l'île, en 2008, de la Porta di Lampedusa-Porta d'Europa, monument du plasticien M. Paladino dédié à la mémoire des migrants disparus en mer : « la signification fondamentale de cette œuvre est de garder en mémoire ces vingt dernières années pendant lesquelles nous avons vu des milliers de migrants mourir en mer, de façon inhumaine, en tentant de rejoindre l'Europe, tragédie sans témoins, souvent sans sépulture et donc sans miséricorde ${ }^{87}$ ». L'île a aussi inventé ses propres rituels de commémoration, après l'échec des funérailles nationales d'octobre 2013. La municipalité a créé un jardin de mémoire, dans la réserve naturelle, en y plaçant 366 arbres (porteurs d'un numéro en attendant de recevoir, un jour peut-être, le nom d'un migrant disparu) et 366 photophores, pour les 366 victimes : pour la maire, c'est « un rite de mémoire et d'espérance (qui) sera le salut de Lampedusa à ces enfants, à nos enfants, mais aussi à tous les naufragés dont on n'a jamais rien su ». Lors du premier anniversaire du naufrage, les gestes symboliques se sont multipliés : gerbes de fleurs jetées en mer, stèle commémorative (marquée de deux mains qui s’étrei-

84. Manfellotto B., « Ora però non dimentichiamoli », L’Espresso, 16 octobre 2013.

85. [http://gatti.blogautore.espresso.repubblica.it/2013/10/30/i-bimbi-che-leuropa-ha-dato-inpasto-ai-pesci/]

86. [http://enigmur.hypotheses.org/3632]

87. Texte de présentation dans les documents de la mairie. Les promoteurs de l'ouvrage sont : Amani Onlus, A. Mosca Mondadori, Alternativa Giovani di Lampedusa, Comunità di Koinonia. Cette sculpture est devenue un emblème des tragédies migratoires en Méditerranée : elle est ainsi reprise en couverture du rapport de l'OIM, Fatal Journeys..., op. cit. 
gnent, et portant les signatures des proches des victimes) déposée sur l'épave sous-marine, «flash mob » des fantômes des défunts surgissant de l'eau, célébration multiconfessionnelle. A la même date, E. De Luca jette sur les lieux du naufrage le «sel de la mémoire » pour que les plaies ne se referment pas, pour que ne soient pas oubliés ceux que les documents de police appellent des « éléments » : «alors il en est ainsi : ici ont coulé les éléments qui forment la chimie humaine de la planète 88 ».

Le réinvestissement des traces laissées par les disparus est aussi un geste fréquent. Le collectif îlien Askavusa a créé Porto M, lieu d'exposition informelle des objets de migrants retrouvés au fil des années sur les côtes de l'île, pour témoigner de l'existence historiquement située de ces migrants inconnus : «(l'objet), de rebut, s'est transformé en stratification de symboles. Il arrive la même chose aux corps des migrants. Ils sont beaucoup représentés dans les médias (...). D’abord, on crée les migrants dans un sens physiologique : ils ont faim, soif, froid, comme des animaux en fuite d'un autre monde. Puis dans les autres sens (politique, culturel, médiatique), en tant que corps qui ne disent rien, muets, sans la possibilité de revendiquer les siècles de colonialisme et d'impérialisme que chacun d'eux porte, consciemment et inconsciemment 89 ». La même inspiration de réinvestissement des traces des disparus, dans une signification plus spirituelle, préside à la réutilisation du bois des épaves, celles des bateaux ayant servi au passage. Un menuisier de l'île a commencé, dès 2009, à fabriquer de petites croix à partir de ce bois : " J'ai eu l'idée d'utiliser le bois des épaves de la décharge pour tailler une croix comme symbole de ce qu'on ne voulait pas faire voir ». Il a aussi fabriqué une grande croix de procession, qui a été bénie par le pape et qui a entrepris, en 2014, un périple mémoriel dans les diocèses italiens, grâce à une association catholique ${ }^{90}$.

Il peut arriver que certains créateurs cherchent à évoquer directement les disparus. Le musicien E. Morricone est allé jusqu'à faire entendre « les voix des noyés », dans une composition donnée à la fin octobre 2013 à Milan, lors

88. De Luca E. [http://fondazionerrideluca.com/lampedusa-1-ottobre-2014/]. Voir aussi la prière laïque pour les morts en mer, qu'il a dite en avril 2015 à la télévision italienne (La Diretta7) [https://www.actualitte.com/article/monde-edition/priere-d-erri-de-luca-auxmigrants-morts-dans-le-naufrage/54963].

89. « Askavusa " signifie " pieds nus " en dialecte sicilien ; [https://portommaremediterraneomigrazionimilitarizzazione.wordpress.com/] ; voir aussi ces images [http://blog.ccfd-terresolidaire.org/npcpca/post/2015/07/26/Lampedusa-lecollectif-Askavusa-la-m\%C3\%A9moire-des-migrants]. En 2015, le plasticien togolais C. Apenouvon a repris la même idée d'exposition des traces des migrants dans son installation «Le film noir de Lampedusa », exposition Visibles/Invisibles, l'Afrique urbaine et ses marges, Fondation Blachère, Apt, France.

90. F. Tuccio, menuisier. On retrouve ici certains des promoteurs catholiques de la Porta di Lampedusa : notamment la Casa dello spirito e delle arti (A. Mosca Mondadori, E. Vai), association catholique qui intervient dans la promotion de la culture, des arts et dans la protection des droits du citoyen, Gandin M., "Il Papa e la croce di Lampedusa », Famiglia cristiana, 9 avril 2014. Voir aussi, sur l'utilisation protestataire des objets, Ritaine E., Le bois des épaves, [http://displacedobjects.com/2015/12/18/le-bois-des-epaves-evelyne-ritaine/]. 
d'un hommage multiconfessionnel 91. Le plasticien danois N. B. Skyum Larsen, après avoir vu les linceuls alignés à Lampedusa, a créé « End of Dreams » : il s'agit de statues dont les formes anthropomorphes évoquent les corps des migrants dans les sacs mortuaires ; réalisées dans un ciment composite qui durcit au contact de l'eau, elles ont été immergées quatre mois au large de la Calabre pour que les mouvements de l'eau les travaillent, puis ensuite exposées dans plusieurs villes européennes 92. Au théâtre, deux œuvres majeures encadrent le naufrage de Lampedusa. L. Prosa, avec « Lampedusa Beach ", créé en 2003, avait déjà mis en scène un (le) naufrage, en montrant sur scène la noyade d'une jeune africaine migrante ; elle a ensuite donné à voir le deuil des familles avec "Lampedusa Way », créé en 2013, pour évoquer la recherche éperdue des parents des disparus, eux-mêmes pris dans le « naufrage horizontal » de la douleur et de la clandestinité : «C'est nous qui faisons naufrage, parce que nous avons perdu les valeurs de fraternité, d'hospitalité 93 », dit-elle. Le texte d'E. Jelinek ${ }^{94}$, écrit lui en 2013-2014 et mis en scène par N. Stemann, «Die Schutzbefohlenen », donne à voir les migrants survivants dispersés en Europe et leur rapport à leurs compagnons disparus. Le texte évoque, avec une mordante ironie, les cercueils d'enfants décorés d'ours en peluche, vus dans le hangar de Lampedusa : "Laissez les petits enfants venir à moi, quand ils réclament leur maman nous les noyons, et sur les cercueils nous mettons un bel ours en peluche, oui, et nous en mettons encore un autre ! Cinq cercueils, cinq ours en peluche ! Cela devrait suffire. Je ne crois pas qu'ils avaient de telles choses, avant. Ils n'avaient pas de cercueils pour jouer, et d'ours en peluche non plus 95 ».

Dans cet ensemble de réactions éthiques et artistiques, les honneurs sous lesquels croule Lampedusa ne peuvent apparaître que comme autant de rituels expiatoires (voir tableau ci-dessous). L'histoire de la proposition de l'île pour le prix Nobel de la paix est, à cet égard, révélatrice. Cette initiative revient souvent en Italie, lors des crises migratoires : ainsi la population du Salento (Pouilles) fut-elle proposée au Nobel de la paix par le quotidien La Gazzetta del Mezzogiorno, en 1999, pour ses efforts humanitaires lors des débarquements des Albanais sur ses côtes ${ }^{96}$. De même S. Berlusconi, alors président du

91. [http://video.repubblica.it/dossier/lampedusa-strage-di-migranti/la-voce-dei-sommersi-1omaggio-di-morricone-alle-vittime-di-lampedusa/144544/143070?ref=nrct-3] ; E. Morricone est lui aussi proche de la Casa dello spirito e delle arti.

92. [http://enigmur.hypotheses.org/3796]; [http://www.nbsl.info/index.html].

93. Lina Prosa, auteure et metteuse en scène italienne, auteure de Tryptique du naufrage [http://enigmur.hypotheses.org/3012] ; Prosa L., Lampedusa Beach suivi de Cassandre on the road et de Programme-Penthésilée : entraînement pour la bataille finale, Paris, Les Solitaires intempestifs, 2012.

94. Elfriede Jelinek, écrivaine autrichienne, prix Nobel de littérature en 2004.

95. Je reprends ici la belle analyse du spectacle faite par Cuttitta P., "Grazie per gli orsacchiotti! Lampedusa e le sue bare, l'Europa e i suoi valori » (traduction : "Merci pour les ours en peluche ! Lampedusa et ses cercueils, l'Europe et ses valeurs »), juin 2014 [http://www.meltingpot.org/Grazie-per-gli-orsacchiotti-Lampedusa-e-le-sue-barel.html\#.VSabnKocQuT] ; voir les images des cercueils [http://palermo.repubblica.it/ cronaca/2013/10/05/foto/lampedusa_le_bare_nell_hangar_della_morte-67969473/1/\#1]. 
Conseil, parla-t-il de présenter Lampedusa à ce même prix, lors de la crise de 2011, durant laquelle des milliers de Tunisiens étaient entassés sur l'île. En 2013, la possibilité d'une candidature a été évoquée, dès le mois d'août, par l'écrivain M. Vargas Llosa (lui-même prix Nobel de littérature) de passage en Sicile, puis par F. Gatti et l'hebdomadaire L'Espresso, qui lance une pétition en ce sens, en octobre: "Lampedusa est officiellement candidate au prix Nobel de la paix 2014. Il s'agit de reconnaître la solidarité manifestée depuis des années par les habitants de l'île, mais aussi la souffrance que les réfugiés survivants doivent affronter et le sacrifice des milliers de personnes mortes noyées ». Quelque chose se clôt, semble-t-il, quand les politiques soutiennent, dans leur propre interprétation, cette initiative. Ainsi, E. Letta, président du Conseil, déclare-t-il : «Lampedusa n'est pas une périphérie de l'Europe : c'est le cœur de notre continent (...). La candidature de Lampedusa au Nobel de la paix est un message d'espérance pour les droits humains et pour la légalité en Méditerranée qui doit mobiliser toute l'Italie et tous les pays de l'UE. Le prix serait une reconnaissance pour une communauté qui enseigne au monde "la globalisation de la solidarité" 97 ».

Bien évidemment, Lampedusa n'a pas obtenu ce prix, et vécu d'autres naufrages. En février 2015, devant les corps de 29 migrants morts de froid en mer, le directeur sanitaire de Lampedusa s'écrit : «Je suis bouleversé, vraiment bouleversé. Je n'arrive pas à m'habituer à ces tragédies. Mais je peux dire que cette fois le drame aurait pu être évité. J'ai le regret de dire qu'avec la fin de Mare Nostrum nous avons recommencé à compter les morts. Je suis fatigué ». Et la lanceuse d'alerte G. Nicolini, la maire de Lampedusa, de conclure : « Les 366 morts de Lampedusa n'ont servi à rien, les paroles du Pape n'ont servi à rien, nous sommes retournés au temps d'avant Mare Nostrum. C'est la réalité 98 ».

Pour ces acteurs directement impliqués, rien ne semble avoir changé. Pourtant, la coalition de cause dont ils font partie exerce une forme de pression politique constante, trouve des alliés à tous les niveaux de gouvernement, et surenchérit à chaque événement migratoire : il en va de même, par exemple, au moment des arrivées liées aux " printemps arabes » ou lors des débarquements dramatiques en mer Egée. De ce point de vue, l'événement Lampedusa semble avoir élargi le recrutement (plus de militants et d'activistes, plus d'intellectuels et de créateurs) et le consensus (affirmation plus forte et plus diversifiée des droits humains des migrants) de cette coalition. Depuis 2013, l'exposition - la visibilité - des naufrages et des morts en mer est notoire et plus

96. Albahari M., 2006, op. cit., p.3.

97. [http://gatti.blogautore.espresso.repubblica.it/2014/02/27/lampedusa-nobel-presentata-lacandidatura/]

98. Docteur P. Bartolo, puis G. Nicolini [http://www.adnkronos.com/fatti/ cronaca/2015/02/09/immigrati-morti-per-ipotermia-profughi-altri-gravissimi _eP8wSAfFHTGuF9rf0s5d6N.html]. 
sujette à débat. Cependant, les événements de 2015, en mer Egée et dans les Balkans, attestent que cent fois sur le métier les militants de la visibilité doivent remettre leur ouvrage.

\section{Les honneurs rendus à Lampedusa 2013-2015}

(liste non exhaustive) $\begin{array}{ll}\text { Octobre } 2013 & \text { Proposition de Lampedusa pour le prix Nobel de la paix (L'Espresso, F. } \\ \text { Gatti) }\end{array}$

Décembre 2013

Le maçon de Lampedusa (qui a participé au sauvetage) est " l'homme de l'année » pour L'Espresso (en couverture)

Mars $2014 \quad$ La RAI offre une vidéothèque à Lampedusa

Mai 2014 Prix «Francesco Arrigoni » (journaliste) aux pêcheurs de Lampedusa

Juillet 2014 Prix «Colombe d'oro per la pace » (Archivio Disarmo, Legacoop) aux pêcheurs de Lampedusa

Juillet $2014 \quad$ Honneur maçonnique « Galileo Galilei » (Grand Orient d'Italie) à la maire de Lampedusa

Juillet $2014 \quad$ Prix Feltrinelli (Accademia dei Lincei) à la population de Lampedusa

Septembre $2014 \quad$ La Fondation Open Society (G. Soros) signe un accord avec la municipalité de Lampedusa (aide au développement socio-territorial)

Prix international " Federichino » pour la solidarité à la commune de Lampedusa

Octobre 2014 (Fondazione Federico II di Palermo, Fondazione di Jesi, Gesellschaft fur staufische Geschichte di Goppingen)

Décembre 2014

Prix «Extravergine » de la solidarité, San Quirico d'Orcia (Fête de I'huile d'olive), à la commune de Lampedusa

Février $2015 \quad$ Médaille d'or de la Croix rouge italienne à la population de Lampedusa $\begin{array}{ll}\text { Février } 2015 & \text { Prix du citoyen européen 2014, Parlement européen, aux citoyens de } \\ \text { Lampedusa }\end{array}$

Août 2015 Prix Rotary «Perdonanza » pour la solidarité 2015 à la commune de Lampedusa

Décembre 2015

Prix pour la paix « Stoccarda » 2015 (Fondazione die AnStifter), à Lampedusa 


\section{Conclusion}

À partir de la singularité d'un événement, le naufrage de migrants le 3 octobre 2013 à Lampedusa, et de ses interprétations, il est possible de comprendre par quels processus l'acceptation tacite d'un état d'exception en mer est rendue possible. Celle-ci dépend du plus ou moins grand degré de visibilité de la mort des migrants. Trois types de cadrage de l'événement s'entremêlent : les migrants noyés sont des corps, des problèmes de politique publique, des personnes dotées de droits. L'irruption des corps migrants, ici l'irruption des morts, fait l'événement : la visibilité de la violence déclenche, par sa diffusion médiatique, de fortes réactions émotionnelles qui ne trouvent jamais vraiment leurs médiations politiques. Les interprétations des responsables de la gestion de la frontière rabattent la visibilité de ces morts à l'intérieur de leurs contextes d'interprétation routiniers : en les « euphémisant », ils en font des ressources argumentatives au sein des controverses entre niveaux de gouvernement pour la prise en charge de la frontière. Les seules interprétations à remettre en cause le cadrage de la politique européenne des frontières sont celles qui affirment les droits humains des migrants en rendant aussi visible que possible leur humanité : elles jouent une partition à long terme qui ne déplace les lignes que très lentement et cherchent en permanence des alliés politiques au sein des institutions.

Les migrants victimes de la mer, eux, malgré les efforts et le talent déployés par ceux qui luttent pour les rendre visibles et respectés, demeurent dans les limbes du politique, hors du cadre, à peine effleurés par les vagues de la crise morale : «La mer est à tout le monde, mais seulement quand on est mort. (...) Réfléchissez, voyez comment l'injustice les poursuit même après la mort. Ils ne sont plus vivants, mais pas morts non plus, étrangers même à cette mince couche de terre qui sépare l'existence de l'inexistence 99 ».

99. Dal Lago A., «Cercueils fluides », Cultures E Conflits, 73, 2009, pp. 109-122, (p. 115). 


\section{Boîte noire méthodologique}

Dans ce travail, il s'agit pour moi de comprendre la portée d'un événement dans le long terme des processus migratoires en Méditerranée. Le défi est de parvenir à suspendre le temps trop rapide de l'événement pour décrire les glissements de sens dans les différentes interprétations des faits.

La recherche prend appui sur la longue durée de mes travaux sur la question migratoire en Italie, menés depuis le début des années 2000. J'ai effectué une observation en temps réel de l'événement du 3 octobre 2013, puis de ses développements entre octobre 2013 et octobre 2014, qui a permis une description précise des acteurs impliqués et de leurs interprétations. L'analyse s'effectue de façon secondaire : lors du déroulement des faits, la saturation du terrain en acteurs divers (notamment l'invasion de l'île par les médias du monde entier) n'aurait pas permis une enquête efficace. Aussi la méthode combine-t-elle revue de presse, analyse des positions et des prises de position des acteurs, observation des mises en scène des événements publics, ainsi que des entretiens de contrôle avec journalistes et chercheurs. En complément, Stéphanie Bonnefille (MCF linguistique cognitive, Climas, Université Bordeaux 3) a effectué une analyse linguistique des principaux discours publics tenus à propos de cet événement.

Outre la presse italienne, ont été étudiés, sous forme de revue de presse systématique, les médias suivants : Le Monde, Libération, La Croix, mediapart.fr, Courrier international, El Pais, The Guardian, The New York Times, Le Devoir, lapresse.ca, ainsi que les informations télévisuelles françaises (journaux télévisés, reportages). Une partie de ces documents peuvent être consultés dans la revue de presse en ligne [http://enigmur.hypotheses.org/2683]. J'ai aussi utilisé l'analyse de la couverture de l'événement par la presse (écrite et télévisuelle) italienne effectuée dans : Osservatorio Carta di Roma, Notizie alla deriva, 2014. Carta di Roma est une association fondée par les organisations de journalistes et par la presse italienne pour veiller à la déontologie de l'information sur l'immigration ; elle est associée à de nombreuses universités italiennes, et pratique une analyse réflexive sur le travail des médias [http://www.cartadiroma.org].

Dans l'écriture des résultats, une des difficultés a été de ne pas pouvoir publier les images diffusées dans la presse. Celles-ci sont au principe même de la portée première de l'événement, celle qui le code en drame humain. Mais leur effet est aussi très labile. Un retour sur les images est donc une façon de montrer à nouveau la violence à l'œuvre. Dans ces images, on retrouve la charge émotionnelle qui marque tant de ces naufrages : le couple enlacé dans l'épave engloutie à Lampedusa en octobre 2013, les ours en peluche sur les cercueils blancs des enfants dans le hangar de Lampedusa, les migrants épuisés sur l'île de Kos durant l'été 2015, l'enfant noyé sur une plage turque en septembre 2015, etc. Lorsqu'une image s'impose par son intensité dramatique, elle parvient à s'extraire du flux continu des informations sur les naufrages qui, par leur récurrence, peuvent créer une perception routinière, un sentiment d'impuissance, et in fine de l'indifférence. Les images remarquables sont, elles, le facteur premier de la visibilité des migrants, morts ou rescapés. Elles imposent cette visibilité à tous les acteurs sociaux et les obligent à répondre à l'émotion et à prendre position. La réflexion sur l'émotion ainsi provoquée par la violence aux frontières montre à quel point le feu de paille émotionnel n'est rien sans un cadrage réflexif et une médiation politique forte. 
Pour pallier ce déficit d'images, dans mon texte, j'ai choisi deux moyens : accentuer les descriptions des situations et donner de nombreux extraits de déclarations; renvoyer le plus souvent possible à des images publiées en ligne, ou sur le carnet de recherches en ligne Enigmur [http://enigmur.hypotheses.org].

$L^{\prime}$ inspiration du thème de cet article m'est venue d'une réaction subjective : depuis longtemps impliquée sur ces questions, et en contact fréquent avec les ONG, j'ai ressenti un profond malaise éthique face à cet énième naufrage. Cette fois, il m'a semblé que je devais dépasser le découragement face à la litanie des morts en Méditerranée et à l'inaboutissement des alertes lancées depuis longtemps par militants, journalistes et experts : le drame humain et l'émotion se sont répétés, presqu'à l'identique, tant de fois, et les chercheurs y sont exposés comme les autres observateurs. J'ai donc tenté de comprendre comment et jusqu'à quel point un fait, à mes yeux insupportable, pouvait être traduit et retraduit presque jusqu'à ne plus faire scandale ou à tomber dans I'oubli. Chercher à élucider les petits arrangements avec la mort (des autres) est alors, pour moi, une façon de continuer à penser, une façon de travailler sur mon émotion, et un moyen de rendre opératoire une révolte éthique : un exercice de réflexivité. 